\title{
Licht- und elektronenmikroskopische Untersuchungen an den Eihüllen des marinen Teleosteers Lutjanus synagris
}

\author{
H. ERHARDT \\ Institut für Zoologie und vergleichende Anatomie der Gesamthochschule Kassel; \\ Kassel, Bundesrepublik Deutschland
}

\begin{abstract}
Light and electron microscopic studies of the envelopes in egg cells of the marine teleost fish Lutjanus synagris. The cortical layer development of the oocytes of the oviparous teleost fish Lutjanus synagris (LINNAEUs) were investigated by light and electron microscopy. In stage I the young oocytes and the follicle cells are in contact with each other. During the early growth period, microvilli cover the surface of the oocytes. In the early stage II, patches of electron dense homogeneous material for the cortex are deposited between the microvilli. In stage III the cortical layer differentiates into a cortex radiatus externus and a cortex radiatus internus. Just before ovulation in stage IV the cortex radiatus externus is strengthened by further differentiations. The transport of material from the egg to the follicle cells and vice versa is probably mediated by pinocytosis. Cytoplasmatic links at the points of contact between microvilli and the branches of the follicular cells could not be observed.
\end{abstract}

\section{EINLEITUNG}

Zur licht- und elektronenmikroskopischen Struktur der Eizellen oviparer Teleosteer liegt seit Jahren eine Anzahl von Arbeiten vor, die viele Gemeinsamkeiten aufzeigen, jedoch auch unterschiedliche Auffassungen erkennen lassen (vgl. ARNDT, 1956, 1960; Flügel, 1967; GötTING, 1961, 1964; MülleR, 1962; WiCKLeR, 1956). So ist die Herkunft der Kortikalschicht auch heute noch recht umstritten, obwohl die Auffassung der meisten Autoren dahin geht, eine oogene Herkunt der Kortikalschicht wahrscheinlich zu machen. Auch die Frage nach dem Stoffaustausch ist noch nicht restlos geklärt, so daß weitere stoffwechselphysiologische Untersuchungen eine eindeutige Beantwortung erbringen müssen.

Speziell bei den Lutjaniden, soweit sie überhaupt bisher untersucht wurden, liegen Besonderheiten gegenüber den bisher untersuchten Teleosteern vor. Kurz vor der Ovulation werden speziell im äußeren Cortex Festigungselemente eingebaut, die zu einer weiteren Differenzierung der Kortikalschicht führen. Dieser Entwicklung sollte durch Untersuchungen an den Eihüllen von Lutjanus synagris (L.) besondere Aufmerksamkeit gewidmet werden. 


\section{MATERIAL UND METHODEN}

Lutjanus synagris erlangt an der kolumbianischen Atlantikküste große wirtschaftliche Bedeutung. Die für die Untersuchung verwendeten Tiere entstammen verschiedenen Fängen, wobei speziell die Bahia Barbacoas, die Ensenada Chengue und das Gebiet vor dem Flughafen Santa Marta als Fangplätze in Betracht kamen. Gefischt wurde mit Handleinen, Fischreusen und Shrimp-Trawls.

Alle Untersuchungen erfolgten an Ovarien, die an Bord den frisch getöteten Tieren entnommen wurden. Teile der beiden Ovarhälften wurden für die lichtmikroskopische Betrachtung in den Gemischen nach Bouin, Susa, in 5\% in 5-bis 10\% /iger Formalinlösung fixiert. Die Einbettung erfolgte über eine steigende Alkoholreihe in Isopropanol-Tissuemat-Gemische in reines Tissuemat. Geschnitten wurde an einem Leitz-Schlittenmikrotom. Die Schnittdicken lagen zwischen $5 \mu \mathrm{m}$ und $10 \mu \mathrm{m}$. Gefärbt wurde fast ausschließlich mit Eisenhämatoxylin nach DeLafield, wobei bemerkt werden muß, daß in vorausgegangenen Untersuchungen sich diese Färbung, neben allen anderen, die ausprobiert wurden, als die geeignetste erwies, die Hüllschichten der Oocyten gut darzustellen. Geeignetes Fixiermittel für die elektronenmikroskopischen. Untersuchungen war das Gemisch nach PALADE (1952). Eine Stückkontrastierung mittels Zusatz von $0,5 \%$ igem Uranylacetat und $1 \%$ iger Phosphorwolframsäure fand bei der Entwässerung im 70\% 0 igem Aceton statt (WoHLfarTHBottermann, 1957). Eingebettet wurde in Vestopal W bei $60^{\circ} \mathrm{C}$ und 48 Stunden. Die Schnitte wurden an einem REICHERT-Ultramikrotom mit Glasmesser hergestellt. Schnittkontrastierungen, soweit sie erforderlich waren, erfolgten mit Bleiacetat nach VENABLE \& Coggeshall (1965). Die Schnitte wurden an einem ZEISS EM $9 \mathrm{~S}$ bei $60 \mathrm{kV}$ untersucht.

\section{LICHTMIKROSKOPISCHE BEFUNDE}

Die Ovarien von Lutjanus synagris liegen im dorsoposterioren Teil der Körperhöhle und füllen diese, je nach Reifegrad der beiden Ovarien, aus. Die stets paarigen Ovarien sind rund und haben tubuläres Aussehen.

Im Verlaufe der Untersuchungen wurden Weibchen mit asymetrischen Ovarien gefunden, die offensichtlich von einer Krankheit befallen waren. Geschlechtsdimorphismus wurde anfangs vermutet, konnte jedoch nicht eindeutig nachgewiesen werden, da das zur Verfügung stehende Material an frühen Reifestadien nicht ausreichend war.

Das Grundschema des Aufbaus der Keimdrüsen ist bei GöTring (1961) zusammengefaßt und diskutiert worden. Es gilt auch für L. synagris. Im Querschnitt gesehen bildet das Ovar nach innen Falten von septenähnlichem Aussehen, in die das Bindegewebe einwächst. In dem in der Mitte verbleibenden Ovarlumen befinden sich bis zu Beginn das Stadiums III Bündel von Blutgefäßen, die offensichtlich Versorgungsfunktionen übernehmen. Sie werden am Ende des Stadiums III zurückgebildet und sind im Stadium IV nicht mehr nachweisbar.

Im Stadium I liegen die Oocyten in Nestern zusammen. Im Stadium II orientieren sie sich zur Peripherie der Einfaltungen hin. Im Stadium III wandern die reifen 


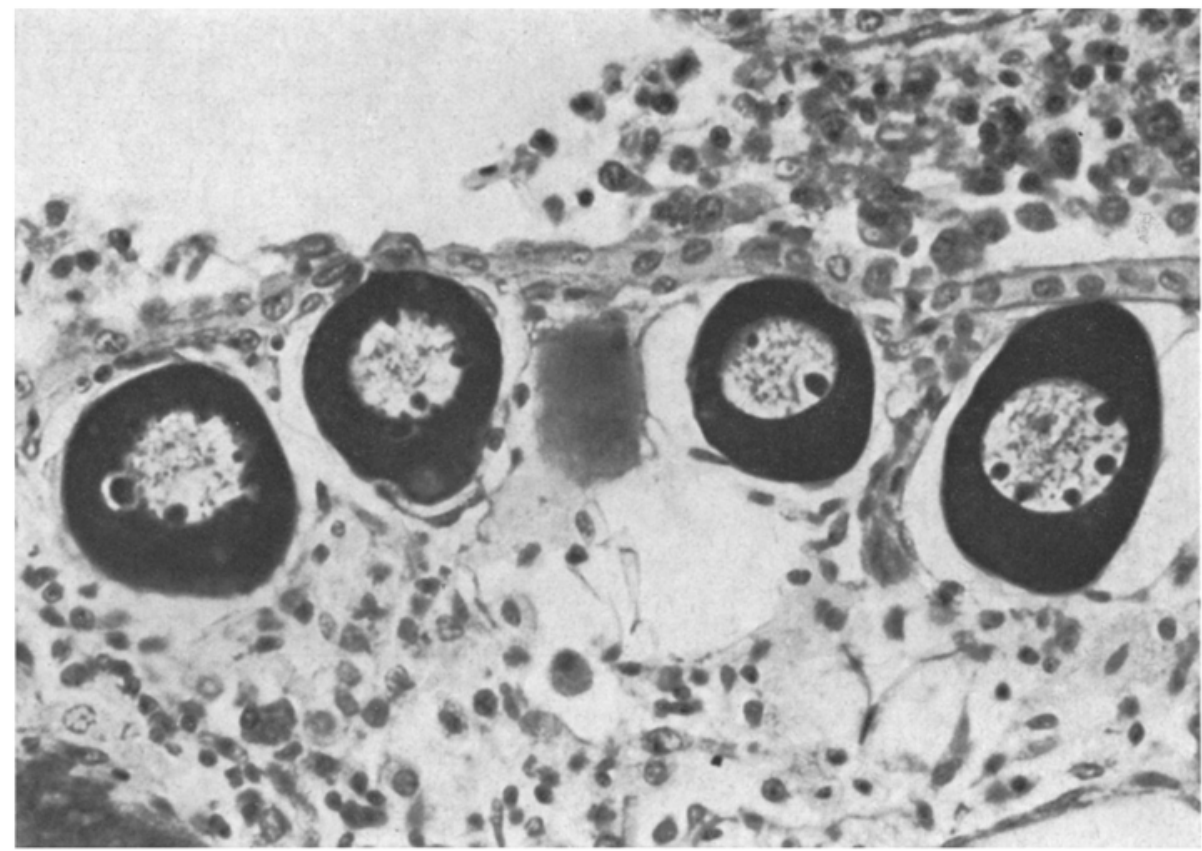

Abb. 1: Oocyten im Ovarstadium I. Die Kerne liegen noch annähernd zentrisch in der Eizelle $(2200: 1)$

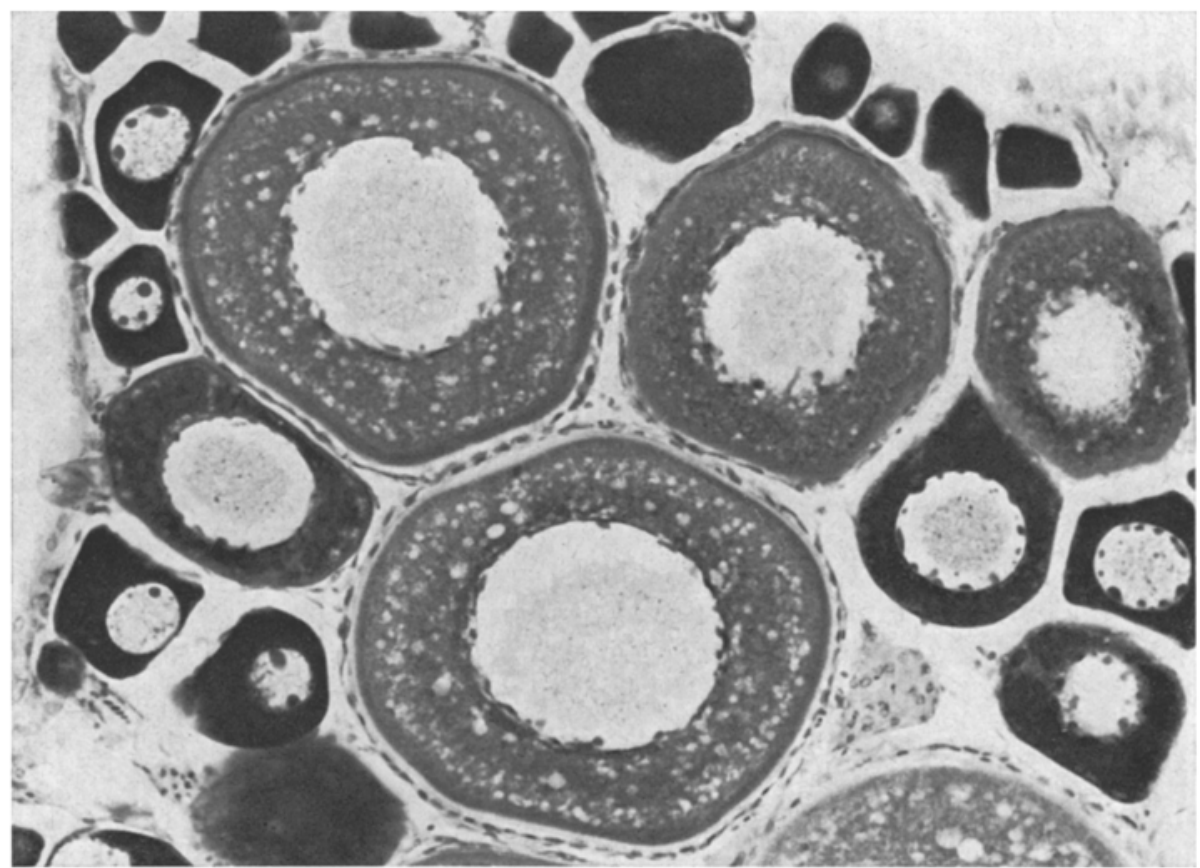

Abb. 2: Oocyten im frühen Stadium III. Ein mehrschichtiges Follikelepithel umgibt die Eizelle $(2350: 1)$ 


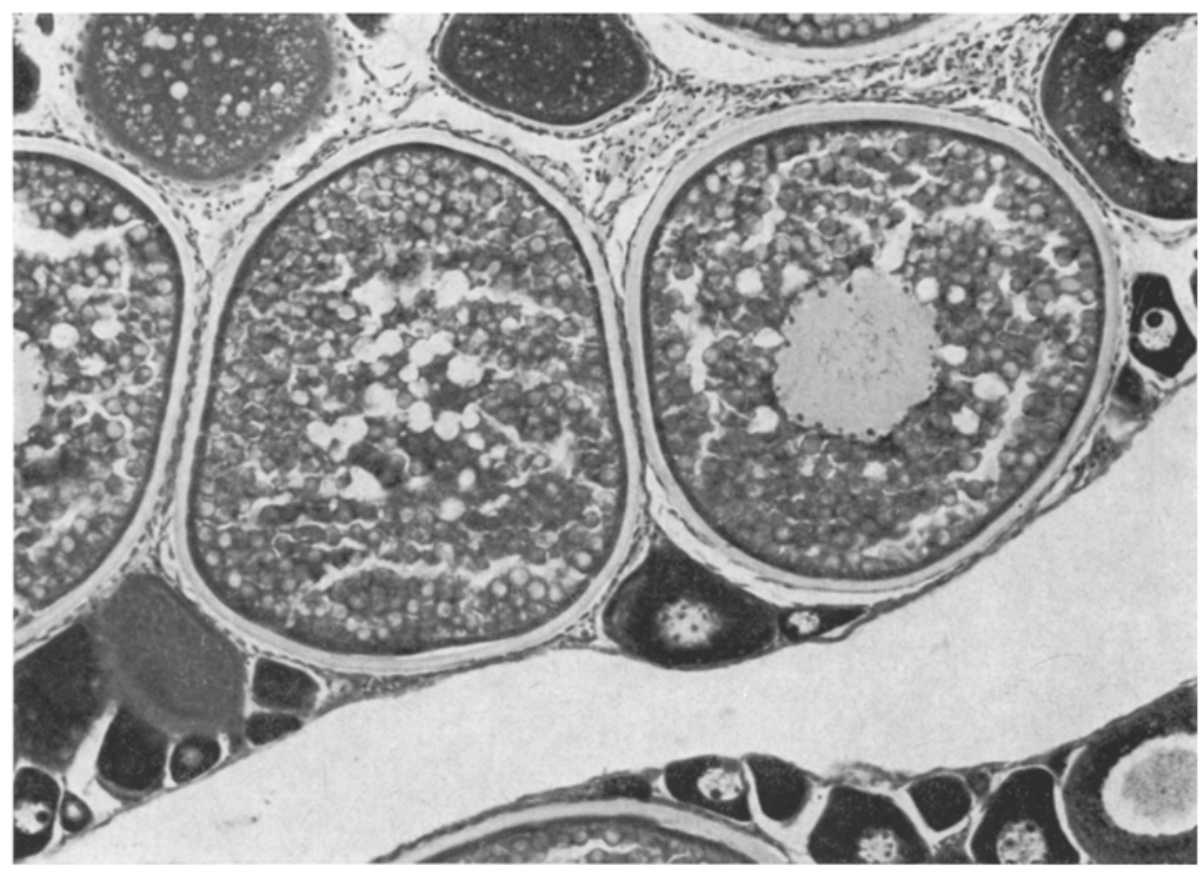

Abb. 3: Oocyten im Stadium IV. Die Eizellen sind mit Dotterpartikeln gefüllt. Die Kortikalschicht ist als dick ausgebildete Eihülle erkennbar $(4750: 1)$

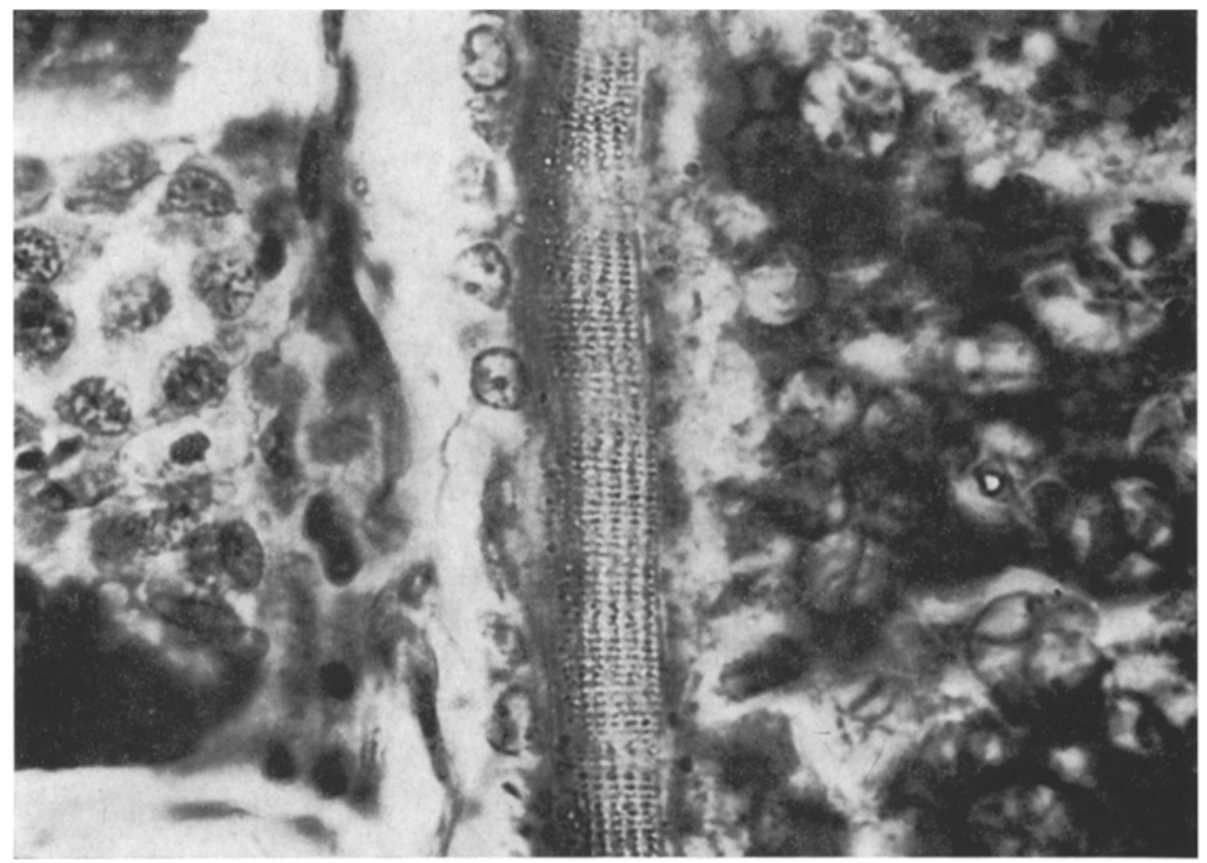

Abb. 4: Die Kortikalschicht einer Eizelle im Stadiun IV. Die krättig ausgebildete Radiärstruktur wird durch die Anordnung der Mikrovilli hervorgerufen. Dicke der Eihülle 2,1 $\mu \mathrm{m}$ $(12500: 1)$ 
Eizellen zur Mitte der Einfaltungen hin, während die Oocyten im Stadium II weiterhin an der Peripherie verharren. Im folgenden sollen die einzelnen Oocytenstadien charakterisiert werden (vgl. Abb. 1-4).

\section{Oocytenstadium I}

Mit Hämatoxylin färbt sich das Oocytoplasma der jungen Eizelle tief blau. Mit dieser und mit anderen üblichen Färbemethoden lassen sich keine weiteren Strukturen im Oocytoplasma nachweisen. Die Oocyten sind konzentrisch gebaut und nehmen bereits in diesem frühen Stadium tropfenförmiges Aussehen an.

Der Zellkern ist im Verhältnis zur gesamten Oocyte groß und blasig. Nicht selten füllt er mehr als $50 \%$ des Volumens der Eizelle aus. Der mittlere Durchmesser der Oocyten beträgt etwa $70 \mu \mathrm{m}$.

Die junge Eizelle wird von einer einfachen Membran umgeben, der primären Oocytenmembran. Sie ist im histologischen Bild sehr schlecht erkennbar, da sie sich in der Färbung kaum vom Oocytoplasma unterscheidet. Unregelmäßig angeordnete Follikelzellkerne, die der jungen Eizelle vereinzelt anliegen, lassen ein einschichtiges Follikelepithel vermuten. Dotterkerne, die am Ende dieses Stadiums auftreten können und die die ersten Anzeichen einer Dotterbildung ankündigen, konnten noch nicht festgestellt werden. Weitere Differenzierungen sind noch nicht erkennbar.

\section{Oocytenstadium II}

Im Stadium II nimmt die Oocyte an Größe und Volumen zu. Ihr mittlerer Durchmesser beträgt $132 \mu \mathrm{m}$. Der Zellkern, der noch immer groß und blasig ist, bleibt im Wachstum gegenüber dem sich vermehrenden Ooplasma zurück. Er enthält mehrere Nucleoli und liegt meist zentral in der Eizelle.

Im frühen Stadium II liegen die Oocyten noch in Nestern zusammen. Mit fortschreitendem Wachstum jedoch lösen sie sich aus ihrer peripheren Position und wandern in die Mitte der Einfaltungen ein. Das Ooplasma ist noch immer intensiv färbbar. Im frühen Stadium II beginnen sich unmittelbar oberhalb der Kernhülle kleine Vakuolen auszubilden, die mit fortschreitendem Wachstum der Eizelle zur primären Oocytenmembran verlagert werden. $\mathrm{Da}$ diese Vakuolenbildung in mehreren Phasen abläuft, kommt es zu einer sichtbaren Schichtung im Ooplasma.

Das zwischen den Vakuolenringen sich befindende Ooplasma erscheint fast homogen. Jetzt beginnt sich ein zusammenhängendes Follikelepithel zu bilden, das mit großer Wahrscheinlichkeit mehrschichtig ist. Die Zellkerne der Follikelzellen sind langgestreckt, nicht selten übereinandergeschichtet und unregelmäßig um die Eizelle angeordnet. Auf den Follikelzellen liegt die Theca folliculi, die vorerst noch keine kontinuierliche Lage bildet.

Neben dem exzentrisch liegenden Zellkern treten die ersten Dotterschollen auf. Sie sind im histologischen Bild als intensiv färbbare Körperchen erkennbar.

Da einige dieser Dotterkörper Ausläufer in das Ooplasma entsenden, ist anzunehmen, daß es sich hierbei um Lipiddotter handelt. Am Ende des Stadiums II be- 
ginnen sich die Zellmembranen einiger Eizellen aufzuwellen. Es muß sich in diesem Falle um frühreife Eier handeln, denn die Aufwellung und die damit verbundene Vergrößerung der Zelloberfläche ist generell ein Merkmal des Stadiums III. Immerhin wird bereits schon hier angedeutet, daß die Eizellen nun in ein Stadium intensiver Wachstumsvorgänge eintreten.

\section{Oocytenstadium III}

Bei einem mittleren Durchmesser von $204 \mu \mathrm{m}$ treten die Oocyten des Stadiums III in eine intensive Wachstumsphase ein. Im frühen III. Stadium sind im Ooplasma zwei oder mehrere Vakuolenringe erkennbar. Dabei wird deutlich, daß die Vakuolen, die dicht unterhalb der primären Oocytenmembran liegen, größer sind als die zentralen.

Der Zellkern bleibt weiterhin im Wachstum gegenüber der sich vergrößernden Eizelle zurück. Die Kernhülle beginnt sich stellenweise aufzuwellen. Im Ooplasma sind nun deutlich Dotterschollen erkennbar, die mit fortschreitender Entwicklung der Eizelle an Größe, Anzahl und Dichte zunehmen. Die primäre Oocytenmembran verdickt sich. Sie wird am Ende des Stadiums III zu einer dicken, gut erkennbaren Kortikalschicht mit einer Dicke von $0,8 \mu \mathrm{m}-1,2 \mu \mathrm{m}$. Obwohl die Kortikalschicht sich stellenweise in ihrer Färbung kaum vom Ooplasma abhebt, sind dennoch Radiärstrukturen in ihr erkennbar. Es ist anzunehmen, daß die Radiärstruktur durch die Anordnung der Mikrovilli hervorgerufen wird, die von der Eizelle in Richtung Follikelepithel und umgekehrt entsandt werden. Die Theca folliculi ist immer noch schmal und dürftig ausgebildet.

\section{Oocytenstadium IV}

Im Stadium IV erfolgt eine weitere Vergrößerung der Oocyten. Der mittlere Durchmesser beträgt $354 \mu \mathrm{m}$. Die im Stadium III noch relativ kleinen Dotterschollen werden größer, wobei sie unregelmäßige Formen annehmen. Die zu Beginn des Stadiums IV noch deutlich vorhandenen Rindenvakuolen verschwinden im Verlaufe des weiteren Wachstums.

Der Nucleus liegt zentral. Er kann sich jedoch kurz vor der Ovulation zur Peripherie der Eizelle verlagern. Der Kern hat nun keine erkennbare Hülle mehr und wird von den umgebenden Dotterschollen ausgebuchtet oder durchsetzt. Die Kortikalschicht hat sich in diesem Stadium zu einer breiten, gut färbbaren Eihülle entwickelt, in der die Radiärstrukturen klar erkennbar werden. Ihr Durchmesser beträgt 2,4 $\mu \mathrm{m}-3,2 \mu \mathrm{m}$.

Das Follikelepithel ist, im Gegensatz zum Stadium III, nicht wesentlich verbreitert worden. Die Theca folliculi bleibt weiterhin dünn und ist oft nicht nachweisbar.

\section{ELEKTRONENMIKROSKOPISCHE BEFUNDE}

Die lichtmikroskopische Einteilung der Oocyten in vier Stadien (ARNDT, 1956) läßt sich auch anhand der sich bildenden und verdickenden Eihülle verfolgen. 


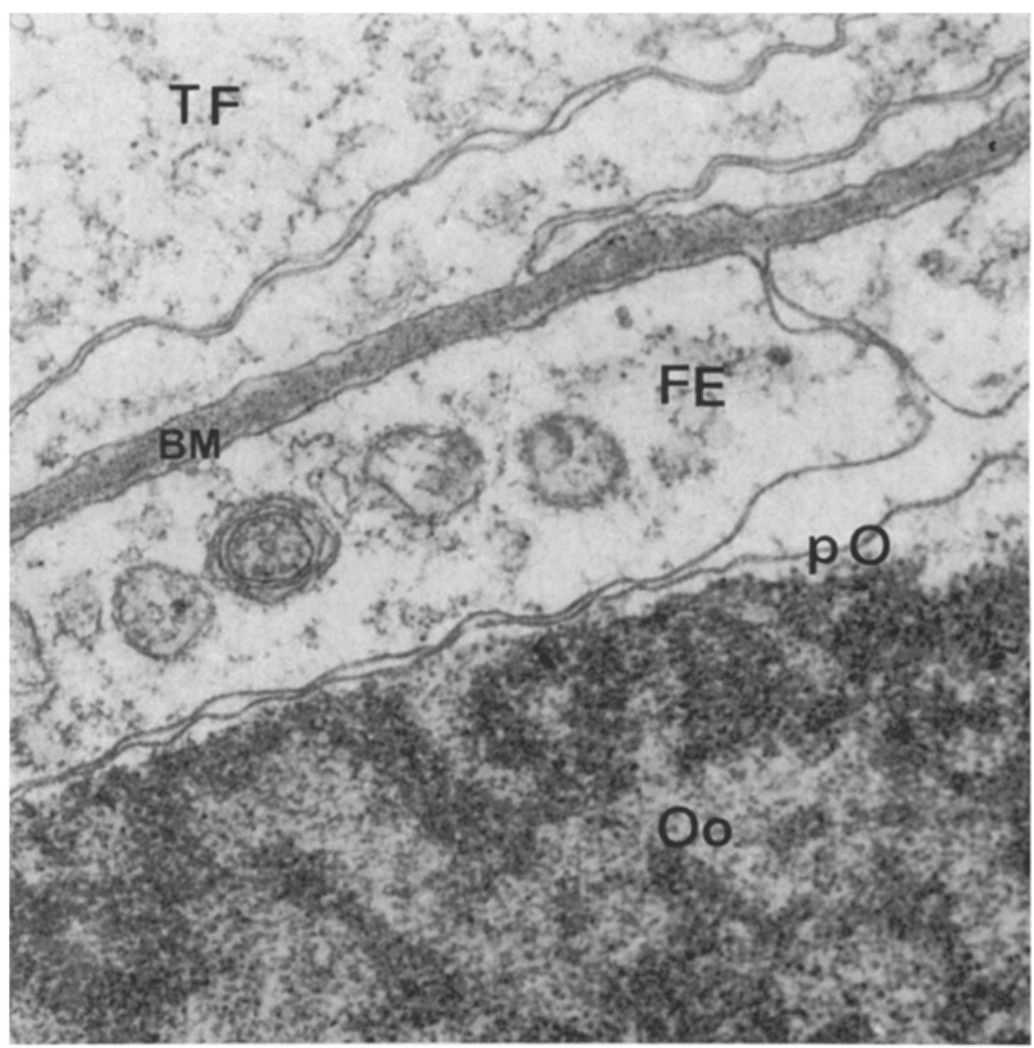

Abb. 5: Oocyte im Stadium I. Follikelepithel und Eizelle sind in sehr engem Kontakt $(112000: 1)$

Erklärungen der Abkürzungen

$\begin{array}{llll}\text { B } & \text { Bündelstrukturen } & \text { MV } & \text { Mikrovilli } \\ \text { BM } & \text { Basalmembran } & \text { MI } & \text { Mitochondrien } \\ \text { Cp } & \text { Caryoplasma } & \text { N } & \text { Nucleus } \\ \text { Crex } & \text { Cortex radiatus externus } & \text { Oo } & \text { Ooplasma } \\ \text { Crin } & \text { Cortex radiatus internus } & \text { P } & \text { Porenkanäle } \\ \text { D } & \text { Dotterschollen } & \text { PO } & \text { primäre Oocytenmembran } \\ \text { FE } & \text { Follikelepithel } & \text { TF } & \text { Theca folliculi } \\ \text { IZ } & \text { Interzellularraum } & \text { V } & \text { Vesikeln }\end{array}$

Bei den Oocyten im Stadium I sind noch keine Differenzierungen ansprechbar, die als Eihülle bezeichnet werden könnten. Die Oocyten dieses Stadiums sind von einer einfachen Elementarmembran umgeben, die als primäre Oocytenmembran bezeichnet wird. Ein einschichtiges Follikelepithel, das sehr reich an Mitochondrien ist, liegt der Oocyte stellenweise glatt auf. Sowohl die Oocyte als auch das Follikelepithel stehen somit in direktem Kontakt miteinander. Erst später beginnen sich beide Membranen aufzuwellen und auseinanderzustreben, so daß der zwischen ihnen liegende Interzel- 


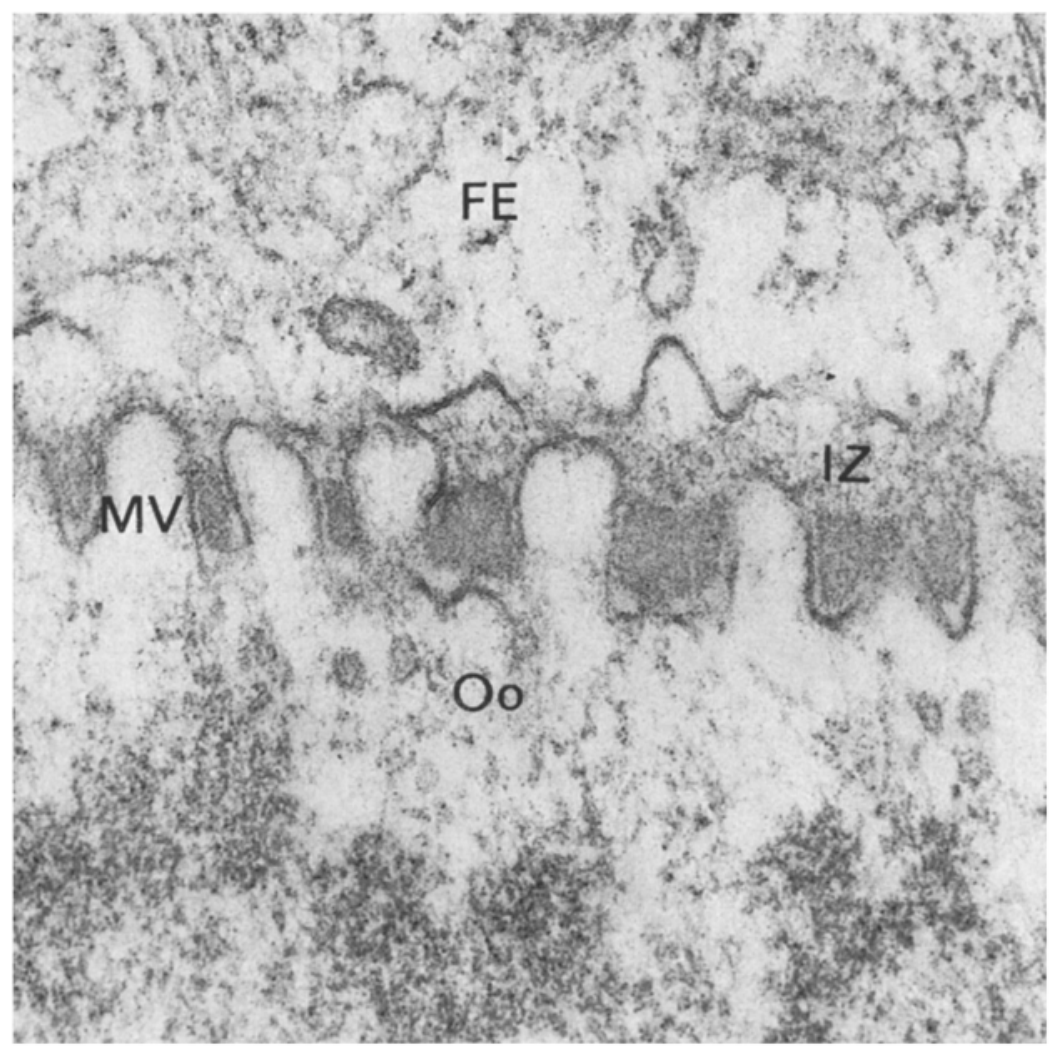

Abb. 6: Oocyte im frühen Stadium II. Elektronenoptisch dichtes Material wird zwischen den Mikrovilli abgelagert $(112000: 1)$

lularraum erweitert wird. Er ist als perioocytärer Raum zu bezeichnen. Dieser ist in diesem Stadium noch frei von jeglichem elektronenoptisch dichtem Material.

Der Zellkern der Follikelzellen ist im Verhältnis zur gesamten Oocyte groß und meistens langgestreckt. Dadurch sind die Zellen arm an Cytoplasma.

Die Eizellen im I. Stadium sind in der Regel oval. Ein großer Nucleus, der nicht selten zwei Drittel der Zelle auszufüllen vermag, liegt konzentrisch in der Eizelle. Er ist reich an Nucleolen, die stets in der Mehrzahl vorhanden sind. Sie bestehen aus einem elektronenoptisch dichten Material und liegen meist peripher im Nucleus. Die Kernhülle ist von Poren durchsetzt. Viele der statischen Bilder sprechen dafür, daß Nucleolenmaterial durch die Kernporen in das Ooplasma der Zellen einwandert, das sich später $z u$ verdichten beginnt (Abb. 5).

Mitochondrien und Vesikeln, die in diesem Stadium verstärkt in der Nähe des Kerns zu finden sind, wandern später zur Peripherie der Eizelle.

Dem Follikelepithel liegt eine einschichtige, sehr dünne Basalmembran auf, die kontrastarm erscheint und stellenweise nur vermutet werden kann. Die sich daran anschließende Theca folliculi ist einschichtig. 




Abb. 7: Oocyte im frühen Stadium III. Die Kortikalschicht ist bereits differenziert $(24500: 1)$

Bereits jetzt, beim Ubergang zum Stadium II, wird häufig Lipiddotter erkennbar, charakteristisch dunkel granulierte Strukturen, die viele Ausläufer in das Oocytoplasma entsenden. Sie sind die Vorboten einer später einsetzenden Dotterbildung. Im Stadium II beginnen sich verstärkt die Membranen sowohl der Eizelle als auch des Follikelepithels aufzuwellen. Der zwischen ihnen entstehende perioocytäre Zwischenraum vergrößert sich zusehends. Es entstehen zuerst kleine, später größere und langgestreckte Fortsätze des Oocytoplasmas, die in den Interzellularraum zwischen Oocyte und Follikelepithel hineinragen. Diese Fortsätze wachsen zu Mikrovilli aus. Erst nachdem sich die Mikrovilli der Oocyte gebildet haben, beginnt das Follikelepithel seinerseits Fortsätze auszubilden, die den perioocytären Raum überbrücken und in Richtung der Oocyte vordringen. Zwischen den Mikrovilli der Oocyte wird eine homogene, elektronenoptisch dichte Substanz abgelagert, die als erste Anlage der Eihülle anzusehen ist. Es konnte nicht geklärt werden, woher das Material stammt, das die Eihülle bildet. Es ist jedoch anzunehmen, daß die Hüllbildung eine Leistung der Eizelle darstellt und nicht, wie auch angenommen werden kann, eine Leistung des Follikelepithels ist. Es wurde beobachtet, daß an den Stellen, an denen Substanz der Oocyte aufgelagert wird, die Oocytenmembran stellenweise aufgelöst erscheint, so daß es durchaus denkbar wäre, daß Material von der Oocyte durch diese Perforationen in den perioocytären Raum gelangen und sich dort zwischen den Mikrovilli der Eizelle ablagern könnte.

Die in diesem Stadium verstärkt einsetzende Mikrovillibildung bedeutet eine 


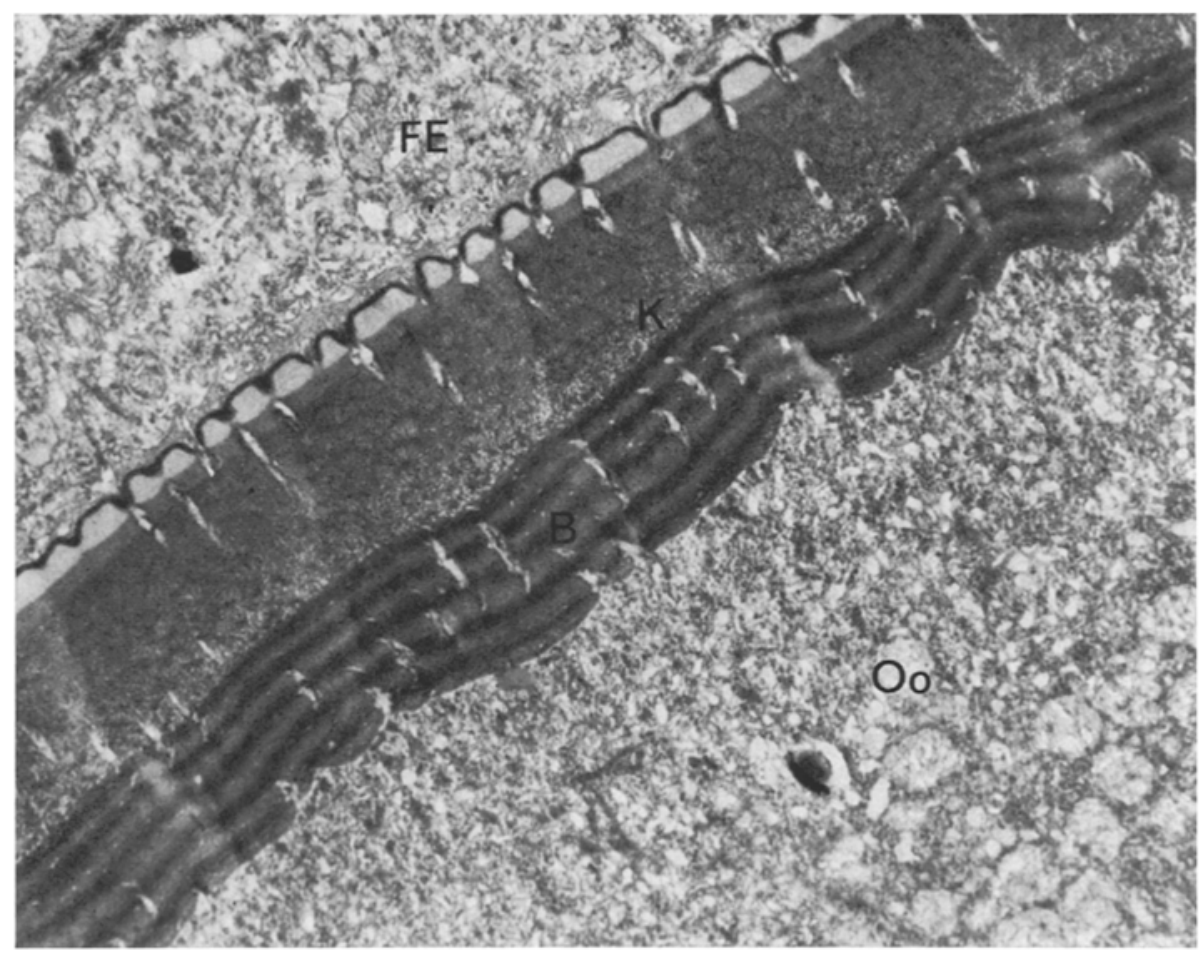

Abb. 8: Oocyte im Stadium III. Weitere Differenzierungen sind in der Eihülle erkennbar $(24500: 1)$

enorme Vergrößerung der Oocytenoberfläche. Die Mikrovilli dringen weit in den Interzellularraum hinein und treten entweder in direkten Kontakt mit den Zellen des Follikelepithels oder mit ihren Ausläufern, den Makrovilli. Die Kontakte erfolgen entweder durch ein Aneinanderlegen beider Mikrovilli oder indem sie sich in Ausbuchtungen der Zelle hineinschieben (Abb. 6).

Auch die Follikelzellen beginnen sich zu vergrößern. Das läßt die Vermutung zu, daß die Oocyte am Ende des II. Stadiums in eine Phase intensiven Wachstums eintritt.

Im Oocytoplasma und im Cytoplasma der Follikelzellen treten zahlreiche Mitochondrien auf. Im Oocytoplasma sind sie zu Bündeln in der Nähe elektronenoptisch dichten Materials lokalisiert, aus denen sich mit großer Wahrscheinlichkeit im weiteren Verlauf des Wachstums die Dotterschollen entwickeln. Vesikeln mit granulären und multifibrillären Einschlüssen treten verstärkt auf. Sie können als Entwicklungsstadien des sich bildenden Dotters gedeutet werden.

Unterhalb der primären Oocytenmembran und im perioocytären Zwischenraum treten kleinere Vesikeln auf, die als Pinocytosebläschen aufzufassen sind.

Im Stadium III nimmt die Oocyte weiterhin an Größe zu. Die Kortikalschicht verdickt sich und erreicht eine Breite von $1,58 \mu \mathrm{m}$. Die vorerst homogene Schicht differenziert sich in deutlich unterscheidbare Abschnitte: in eine äußere helle Schicht, 




Abb. 9: Zwei nebeneinanderliegende Oocyten im Stadium III $(5400: 1)$ 




Abb. 10: Die Kortikalschicht einer Oocyte im Stadium IV. Porenkanäle sehr schmal und langgestreckt $(20520: 1)$ 
den Cortex radiatus externus, und in eine elektronenoptisch dunklere Schicht, den Cortex radiatus internus. Beide Schichten können am Ende des III. Stadiums weitere Differenzierungen erfahren, die jedoch erst für das folgende IV. Stadium charakteristisch sind. Die Abbildungen 7 bis 9 zeigen verschiedene Aspekte der Feinstruktur von Oocyten des Stadiums III.

Die Kortikalschicht wird von Poren durchbrochen, durch die sowohl die Mikrovilli der Oocyte als auch die des Follikelepithels verlaufen. Schräg angeschnittene Eihüllen zeigen, daß in den Porenkanälen meist zwei Mikrovilli verlaufen. Zahlreiche Dotterschollen unterschiedlicher Größe sind im gesamten Oocytoplasma verteilt. Abgeschnürte Vesikeln des Dalton-Komplexes sind sehr häufig in der Nähe von Membranen zu finden. Es ist anzunehmen, daß sie membranöse Anteile für die sich vergrößernde Eizelle liefern.

Der Nucleus ist im Verhältnis zur gesamten Zelle kleiner geworden. Er liegt nun zentral. Die Kernhülle ist stark aufgewellt und läßt fingerförmige Einstülpungen in das Caryoplasma erkennen. Nucleolen sind noch immer in der Mehrzahl vorhanden. Das Follikelepithel hat sich verbreitert.

Die Oocyten des Stadiums IV stehen kurz vor der Ovulation. Die Kortikalschicht hat nun einen Durchmesser von $5,99 \mu \mathrm{m}$. Sie wird weiterhin von Porenkanälen durchbrochen, in denen sehr dünne und langgestreckte Mikrovilli verlaufen (Abb. 10).

Die Porenkanäle werden, kurz bevor die Eizelle in ihre planktische Phase eintritt, mit großer Wahrscheinlichkeit geschlossen.

Die Kortikalschicht hat sich nun weiter differenziert. Der Cortex radiatus externus, der im Stadium III noch keine sehr deutlichen Differenzierungen aufwies, läßt drei radiäre Schichten erkennen. Den äußeren Abschluß des fertigen Eies stellt eine sehr dunkel granulierte Schicht dar. Zwei darunter liegende Schichten unterschiedlicher Granulierung folgen. Der sich daran anschließende Cortex radiatus internus dagegen besteht, wie bei den meisten Teleosteern, aus einer äußeren homogenen Schicht mit fibrillären Mustern und einer weiteren, sich von ihr gut differenzierenden Lage, in der sechs konzentrische Bündelstrukturen verlaufen.

Die Oocyten dieses Stadiums sind erfüllt von Dotterschollen unterschiedlicher Größe und Granulierung. Sie reichen bis dicht an die primäre Oocytenmembran. Das Ooplasma ist weitgehend verdrängt worden. Rindenvakuolen und Vesikeln, die im Stadium III noch vorhanden waren, verschwinden weitgehend. Das Follikelepithel ist zurückgebildet, was darauf hinweist, daß der Stoffaustausch weitgehend abgeschlossen ist.

\section{DISKUSSION}

Die licht- und elektronenmikroskopischen Untersuchungen sollten Aufschluß darüber geben, wie sich die Oocyten mit fortschreitender Reife entwickeln und welche Besonderheiten speziell bei der Bildung der Eihüllen auftreten.

Schon die lichtmikroskopisch sichtbare Radiärstreifung der Kortikalschicht wird, wie elektronenoptische Befunde belegen, durch radiär verlaufende Porenkanäle hervorgerufen. Nachdem frühere Autoren die Auffassung vertraten, die Kortikalschicht sei eine Leistung des Follikelepithels (CHAUdHRY, 1956; WiCKLER, 1957), konnten SPECK (1933), ARndt (1956, 1960), GötTing (1970) und andere Autoren die oogene Her- 
kunft der Kortikalschicht der Teleosteer-Oocyten wahrscheinlich machen. Nach der heutigen Auffassung der meisten Autoren ist sie eindeutig eine Differenzierung der Eizelle selbst.

Das Schicksal der primären Oocytenmembran während des Prozesses der Eihüllbildung war lange Gegenstand unterschiedlicher Auffassungen. Widersprüchliche Meinungen bestanden darüber, ob die homogene Schicht, die zwischen den Mikrovilli der Oocyte abgelagert wird und aus der sich die Kortikalschicht zu bilden beginnt, innerhalb oder außerhalb der primären Oocytenmembran liegt. Heute ist es unumstritten, daß die Kortikalschicht der primären Oocytenmembran aufgelagert wird und somit außerhalb der Eizelle liegt.

Die weitere Unterteilung der Kortikalschicht in einen Cortex radiatus externus und internus, wie sie von Görtring (1964) vorgenommen wurde, beschreibt lediglich unterschiedliche Strukturierungen der sich bildenden und verdickenden Eihülle. Weitere Differenzierungen innerhalb der von GöTTING getroffenen Unterteilungen, die in diesen Untersuchungen festgestellt wurden, weisen darauf hin, daß zusätzliche Stützelemente vor allem in den Cortex radiatus externus vor der Eiablage eingebaut werden, die es der Eizelle ermöglichen, relativ große physikalische Beanspruchungen während ihrer planktischen Phase zu ertragen. Als mikromorphologisch auffällige Kontaktstrukturen zwischen der Eizelle und dem sie umgebenden Follikelepithel fungieren die Mikrovilli der Eizelle und die Ausläufer des Follikelepithels. Während die Mikrovilli der Oocyte durch die sich im Verlaufe des Wachstums verdickende Kortikalschicht verlaufen und in den perioocytären Raum zwischen Eizelle und Follikelepithel hineinragen, sendet das Follikelepithel seinerseits Fortsätze in Richtung der Oocyte aus. Zuerst in diesem Interzellularraum, später in den Porenkanälen der Kortikalschicht treten die Zellmembranen der Mikrovilli der Eizelle und die der Ausläufer des Follikelepithels in engen Kontakt miteinander.

Eine Auflösung der Zellmembranen an den Kontaktstellen konnte in keinem Falle beobachtet werden.

Yamomoto \& Onazato (1965) und Jollie \& Jollie (1964) fanden bei Süßwasserfischen Desmosomen als Kontaktstrukturen bei jungen Eizellen. Das kann bei Lutjanus synagris nicht bestätigt werden. Eine eindeutige Beantwortung der Frage nach dem Ablauf des Stoffwechsels, der zwischen der Eizelle und dem Follikel erfolgt, muß noch ausbleiben. Wie MüLler (1961) und FlüGEL (1967) vermuten, enthält der perioocytäre Raum zwischen Eizelle und Follikel Lösungsprodukte, die aus dem Follikelepithel stammen und die von den Mikrovilli der Eizelle resorbiert werden. Andererseits müssen auch die Stoffwechselprodukte, die sich für die weitere Entwicklung der Eizelle als schädlich erweisen, entweder direkt an das Follikelepithel oder in den Interzellularraum abgegeben werden.

$\mathrm{Da}$, wie bereits erwähnt, kein Fusionieren der Zellmembranen an den Kontaktstellen beobachtet werden konnte, liegt die Vermutung nahe, daß ein anderer Transport existiert als der, der durch den direkten Kontakt der beiden Mikrovilliplasmen möglich ist. Es darf jedoch das Vorhandensein einer zeitlich begrenzten cytoplasmatischen Einheit beider Ausläufer nicht von vornherein ausgeschlossen werden, denn immerhin vermittelt das elektronenmikroskopische Bild nur einen statischen Ausdruck ständig ablaufender dynamischer Prozesse. 
Ein Stofftransport mit dem Mechanismus der Mikropinocytose, wie er von Jollie \& Jollie (1964) für Lebistes reticulatus beschrieben wurde, kann auch bei Lutjanus synagris angenommen werden, da viele kleine Vesikeln und pinocytotische Bläschen in allen Zellschichten nachgewiesen werden konnten.

\section{ZUSAMMENFASSUNG}

1. Die Struktur der Eier und Eihüllen reifender Oocyten von Lutjanus synagris (L.) wurde licht- und elektronenmikroskopisch untersucht.

2. Die Bildung der Kortikalschicht beginnt im frühen Stadium II. Eine elektronenoptisch dichte Substanz wird zwischen den Mikrovilli der Oocyte abgelagert.

3. Im frühen Stadium III differenziert sich die Kortikalschicht in einen Cortex radiatus externus und einen Cortex radiatus internus.

4. Im Stadium IV wird der Cortex radiatus externus weiter differenziert: Er besteht aus drei deutlich unterscheidbaren Lagen, einer sehr dunklen Außenlage und zwei darunterliegenden Schichten unterschiedlicher Granulierung.

5. Die Kortikalschicht wird von Poren durchbrochen, in denen die Mikrovilli der Oocyte und die Fortsätze des Follikelepithels verlaufen.

6. Der Stofftransport von der Eizelle zum Follikel und umgekehrt erfolgt mit großer Wahrscheinlichkeit auf dem Wege der Pinocytose.

7. Cytoplasmatische Verbindungen an den Kontaktstellen zwischen den Mikrovilli der Eizelle und den Fortsätzen des Follikelepithels konnten nicht beobachtet werden.

Anmerkung. Die vorliegende Arbeit stellt einen Teil der Inaugural-Dissertation zur Erlangung des Doktorgrades an der Naturwissenschaflich-Philosophischen Fakultät der Universität Gießen dar.

\section{ZITIERTE LITERATUR}

ARndt, E. A., 1956. Histologische und histochemische Untersuchungen über die Oogenese und bipolare Differenzierung von Süßwasser-Teleosteern. Protoplasma 47, 1-36.

- 1960. Untersuchungen über die Eihüllen von Cypriniden. Z. Zellforsch. mikrosk. Anat. 52, 315-327.

Chaudhry, H. S., 1956. The origin and structure of the zona pellucida in the ovarian egg of teleost. Z. Zellforsch. mikrosk. Anat. 43, 478-485.

FLÜGEL, H., 1967. Elektronenmikroskopische Untersuchungen an Oocyten und Eiern des Flußbarsches Perca fluviatilis. Z. Zellforsch. tnik rosk. Anat. 77, 244-256.

- 1970. Licht- und elektronenmikroskopische Untersuchungen an Oocyten und Eiern einiger Knochenfische. Z. Zellforsch. mikrosk. Anat. 83, 82-116.

Görrting, K. J., 1961. Beiträge zur Kenntnis der Grundlagen der Fortpflanzung und zur Fruchtbarkeitsbestimmung bei marinen Teleosteern. Helgoländer wiss. Meeresunters. 8, $1-41$.

- 1964. Entwicklung, Bau und Bedeutung der Eihüllen des Steinpickers (Agonus catapbractus L.). Helgoländer wiss. Meeresunters. 11, 1-12.

Jollie, W. P. \& Jollie, L. G., 1964. The fine structure of the ovarian follicle of the ovoviviparous poeciliid fish Lebistes reticulatus. J. Morph. 114, 503-526. 
MülLER, H., 1962. Elektronenmikroskopische Untersuchungen zur Bildung der Eihüllen bei Zahnkarpfen. Verh. dt. zool. Ges. (Zool. Anz. Suppl.) 25, 294-306.

Speck, J., 1933. Die dipolare Differenzierung des Protoplasmas des Teleosteereies und ihre Entstehung. Protoplasma 18, 497-545.

WICKLER, W., 1956. Der Haftapparat einiger Cỉchliden-Eier. Z. Zellforsch. mikrosk. Anat. 45, 304-327.

Anschrift des Autors: Dr. H. ERHARdT

Institut für Zoologie und vergleichende Anatomie

Gesamthochschule Kassel

Heinrich-Plett-Str. 40

D-3500 Kassel

Bundesrepublik Deutschland 